\title{
Reality Simulation for Bike Training Devices with Touch Panel
}

\author{
Ter-Feng Wu, ${ }^{1 *}$ Pu-Sheng Tsai, ${ }^{2}$ Nien-Tsu Hu, ${ }^{3}$ Jen-Yang Chen, ${ }^{4}$ and Yi-Shun Huang ${ }^{2}$ \\ ${ }^{1}$ Department of Electrical Engineering, National Ilan University, Yilan 26047, Taiwan, R.O.C. \\ ${ }^{2}$ Department of Electronic Engineering, China University of Science and Technology, Taipei 11581, Taiwan, R.O.C. \\ ${ }^{3}$ Chemical Systems Research Division, National Chung-Shan Institute of Science and Technology, \\ Taoyuan 32599, Taiwan, R.O.C. \\ ${ }^{4}$ Department of Electronic Engineering, Ming Chuan University, Taoyuan 33348, Taiwan, R.O.C.
}

(Received October 26, 2017; accepted December 5, 2017)

Keywords: bike reality simulation, real-time clock, RFID, touch panel

In this work, we developed a stationary bike reality simulation training device. The core of the system is an ATMega 162 microcontroller, produced by Atmel, which is combined with a real-time clock (DS1287), a $16 \times 4$ backlight liquid crystal display (LCD) module, an ICL232 level shifter chip, and a radio frequency identification device (RFID) card and its reader. The system is operated in coordination with a graphic control interface in Chinese, designed on Weintek's touch panel, to create an operational simulation environment. Users can select the cycle path using a human-machine interface. The stationary bike assistive training device that is proposed in this paper helps riders undertake simulation-based training before cycling along a real cycle path. They can cycle along the real path after satisfying certain criteria and passing a test. When the wheels of the bike are revolving, a Hall sensor and our magnets generate pulse signals. A memory control unit (MCU) is used to read pulse signals, calculate riding speed, and accumulate the mileage and time ridden. Each riding record, including mileage, duration, average speed, and the path chosen, will be recorded in the RFID card. When the riding database is turned on, the data in the memory of the RFID card are read automatically; the last eight training records are displayed on the touch panel.

\section{Introduction}

In recent years, city populations have reached saturation, causing excessive loading of public transportation systems. As global warming accelerates, environmental issues become more popular, energy prices keep reaching new highs, and large populations are moving from the countryside into cities all over the world. People are demanding alternative transportation for leisure purposes, such as picnicking and short trips. Meanwhile, environmental protection and energy saving are becoming increasingly urgent. Bicycles, a pollution-free form of transportation, are naturally attracting attention. Population and social trends are contributing to the rapid development of the bicycle market. Populations are aging as a result of falling fertility and improved medicine and healthcare. Bicycles, which are convenient and good for

*Corresponding author: e-mail: tfwu@niu.edu.tw

http://dx.doi.org/10.18494/SAM.2018.1787 
fitness, have recently become extremely popular because they save petrol, are emission-free, make no noise, and consume no power.

The stationary bike simulation-based training device ${ }^{(1-4)}$ in this work turns people's imagination into real life. When the wheels of the bike are revolving, a Hall sensor and our magnets generate pulse signals. A memory control unit (MCU) is used to read pulse signals, calculate riding speed, and accumulate the mileage and time ridden; these are the basis of the bicycle odometer. Moreover, the landscape on site and the exercise status are reflected in animation. The system provides recreation for those who live at high latitudes or in metropolitan areas and often cannot enjoy riding outside because of poor weather, such as snow in winter, a crowded living environment, and issues of road traffic safety. Currently, most virtual reality ${ }^{(5-11)}$ training platforms for bicycles are produced by European and American manufacturers, such as Kinetic, Elite, and Tacx, because they are mostly provided to bicycle riders who train daily for races, and as a result of this, virtual reality technologies and their related products are highly expensive. If the cost of the whole set of training device can be reduced and the local elements of Taiwan are added at the same time, it is considered that visibility and acceptability of the bicycle virtual reality training device will be enhanced. By doing this, we expect to increase the added value of the bicycle industry in Taiwan, promote the development of relevant industries, and deepen the impact of energy saving and carbon reduction that the bicycle industry brings to green cities.

Manufacturers that have developed virtual reality training platforms include Tacx from Holland, which offers the Tacx virtual reality training station. The most attractive feature of this product is that it reflects various environmental conditions in the experience of riding . For example, the fly wheel drive of the microcontroller in the system generates tremendous resistance at low speed when climbing, providing the sense of climbing a hill or a mountain. When going downhill, the microcontroller of the training station actively drives the rear wheels of the bicycle, making the ride very fast.

\section{System Architecture of Stationary Bike Training Device}

In this work, a "stationary bike reality simulation training device" is designed to enable riders to enjoy training indoors. The designed training platform not only simulates riding outdoors, but more importantly, the simulation-based trainer provides riders with an index of physical training to which competitive riders can refer. Competitions, such as the Sun Moon Lake Triathlon, and various challenging cycle paths, such as the Huatung Provincial Highway No. 9, can be simulated. Figure 1 shows the system architecture, which can be divided into three subsystems namely, (1) the odometer, (2) the radio frequency identification device (RFID) card reader, and (3) the reality simulation design of a touch panel.

The goals of this work include the following. (1) Design a wheel revolution measurement system with a Hall sensor circuit and four magnets and installing it on the front revolving axle of a stationary bike; owing to the characteristics of the Hall circuit, the wheel generates four pulses per revolution. (2) Use an ATMega 162 microcontroller, produced by Atmel, as the core chip; combine it with the RTC DS1287 chip and the ICL232 level shifter chip to develop 


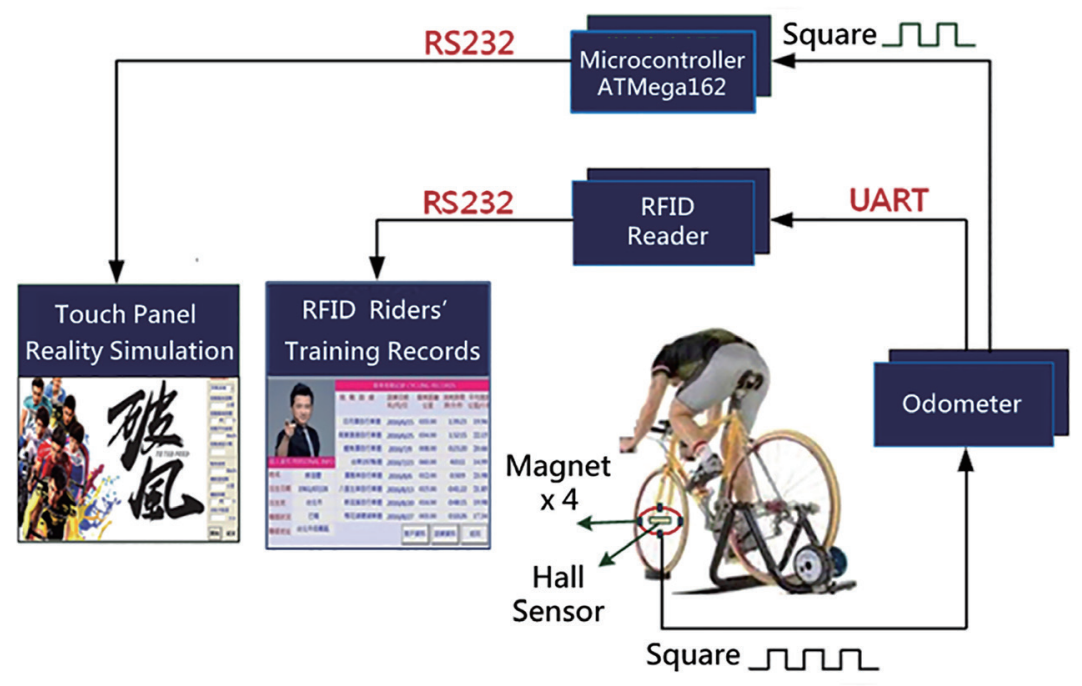

Fig. 1. (Color) Block diagram of system.

a bicycle odometer. Riding information can be displayed during training; the current time, exercise duration, accumulated mileage, and average riding speed are displayed. (3) Design something similar to the membership card of a fitness center with RFID and record riding information for each session, including the kilometer cycled, exercise time, average speed, and path cycled; all this information can be recorded in the RFID card. Establish an "RFID riders training database" for the working platform, using the graphic control interface that is designed on Weintek's touch panel; the information on the RFID memory card can be read automatically and the last eight training records will be displayed on the touch panel. The RFID card can also save personal fitness information to enable training effectiveness to be evaluated. (4) Design a window environment of "reality simulation" in the graphic control interface on Weintek's touch panel; riders can choose among eight classic cycling paths in Taiwan. To simulate reality, the screen shows the local scenery and landscape along the chosen path. The speed by which the scene and objects along the path go by varies with the speed of the bicycle, providing some visual enjoyment to riders during an otherwise boring training process. The software also provides total kilometers traveled, time left, and average speed along each path as reference indices using which riders on the real path can judge their success.

\subsection{Odometer design}

Figure 2 shows the hardware circuit of the odometer that is designed in this work. The overall architecture uses an ATMega 162 microcontroller as its core, which is combined with a DS1287 real-time clock rate chip for clock display, an ICL232 level shifter chip to convert the UART signal into an RS232 signal (level: check), and a $16 \times 4$ backlight liquid crystal display (LCD) module to display information on the odometer. As shown in Fig. 3, information about calories consumed and physical fitness can be estimated from the weights of riders and all this information can be transmitted by Bluetooth for display on a smartphone, as shown in Fig. 4. 


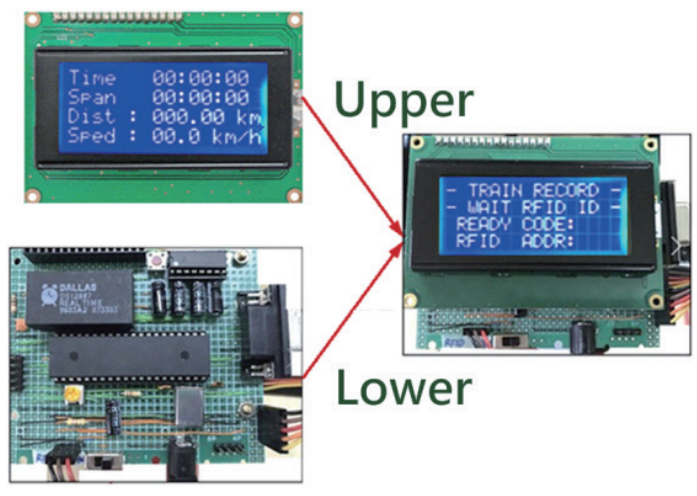

Fig. 2. (Color online) Combination of upper and lower layers.

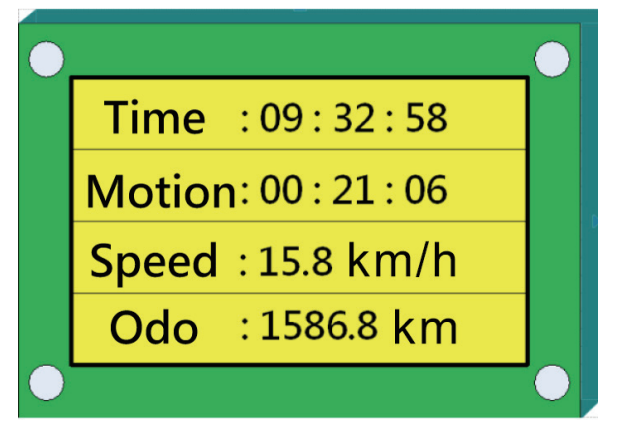

Fig. 3. (Color online) Information displayed on odometer.

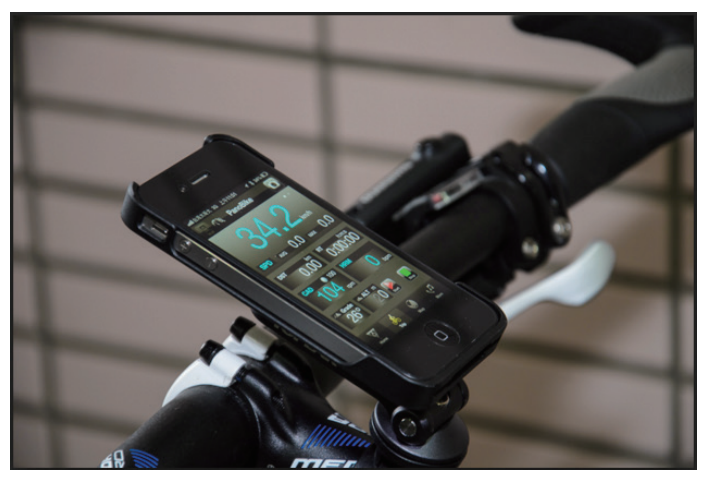

Fig. 4. (Color online) Mileage information displayed on smartphone.

\subsection{Frequency identification}

The soul of the system is RFID. It contains a reader and RFID tag, and the principle of its operation is to use the sensor to transmit radio waves and then trigger the RFID tag within the range of sensing. An electric current is generated by electromagnetic induction to supply the chip on the RFID tag and to transmit electric waves in response to the sensor. The most common tag is the Easy Card. A readable and rewritable RFID module that is produced by Parallax Company is used in the system herein. Figure 5 shows this module; on the left is an RFID sensor in the middle is an RFID pin line, and on the right is an RFID tag.

The main purpose of RFID is to save various motion parameters after training as a basis for evaluating physical fitness. Weintek's touch panel MT6071iE is used to create a "stationary spinning bike reality simulation training database" on the RFID card for the human-machine interface working platform, which stores motion parameters for riders who have completed the training. These parameters include total mileage, duration of training, average speed, and paths cycled. Figure 6 shows the RFID rider training database. A user who puts his or her personal card on the RFID card reader can read the information in the memory of the card. RS232 serial communication is used to transmit this information to the touch panel. During the data transmission process, a flashing icon appears in the lower right-hand corner of the screen; personal photographs, personal data, and riding records can all be transmitted. We hope that 

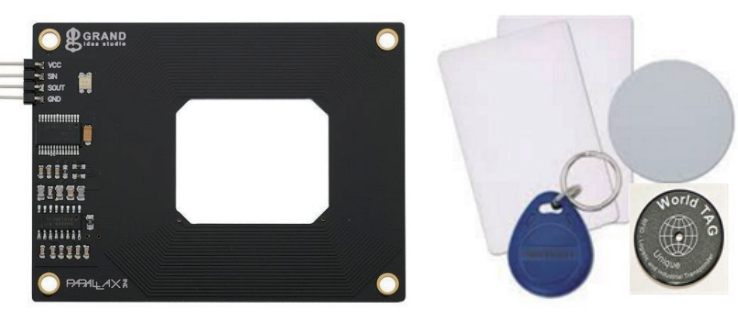

Fig. 5. (Color online) RFID produced by Parallax.

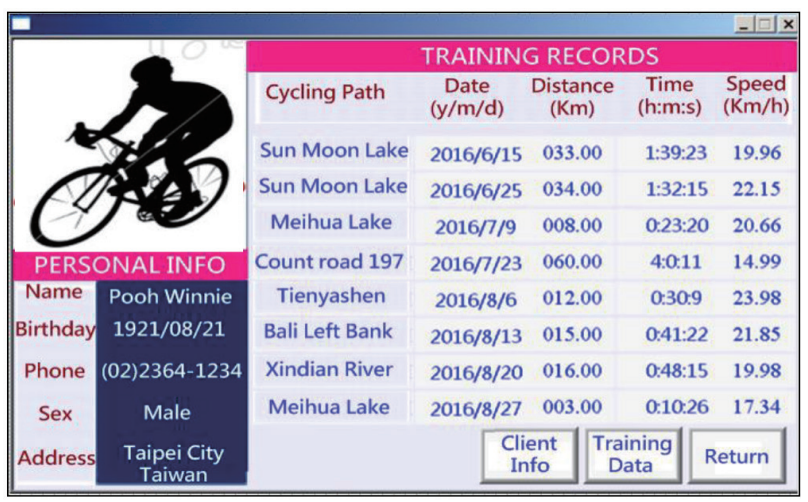

Fig. 6. (Color online) Image of RFID rider training database.

the system can be combined with the fitness passport that the Ministry of Education is currently promoting. Physical fitness can be seen as the body's adaptability to a dynamic environment, which may involve climate change, viral infection, physical labor, or exercise and, like regular exercise that supports it, is very important.

\subsection{Stationary spinning bike reality simulation}

In this work, we developed a window-based display and a program to control a simulation of reality. A serial communication component, MSComm, in the graphic toolbox of Weintek and the control element, Timer, are used to calculate a rider's average speed, accumulated mileage, and exercise duration from the number of pulses that MSComm receives from the wheel revolution measurement system in every sampling period. The screen displays this information. To add the elements of localization, eight classic cycling paths in Taiwan are provided. They are (1) Sun Moon Lake Cycle Path, (2) Hsinchu Nanliao Fishing Port Cycle Path, (3) Liyutien Cycle Path, (4) Taitung Chishang County Highway No. 197, (5) Tienyashen Cycle Path, (6) Bali Left Bank Cycle Path, (7) Xindian River Cycle Path, and (8) Meihua Lake Round-the-Lake Cycle Path.

Riders can select any of the above paths, and the simulation screen will provide a brief introduction to it. If users select (4) Taitung Chishang County Highway No. 197, then Fig. 7 will be displayed on the screen. The right-hand side of the message column indicates that the total length of the path is $60 \mathrm{~km}$; the mean speed is $15 \mathrm{~km} / \mathrm{h}$, and the total riding time is $4 \mathrm{~h}$. When a rider is ready to train, he or she presses "Start" and the window screen provides images of County Highway No. 197. In this project, the control screen is from a first person perspective; therefore, the rider sees all of the scenery along the road to start to move backward slowly, and the speed of this backward motion is directly proportional to the riding speed. Riders can train while enjoying the beautiful views.

The window-based reality simulation software provides 100 images of the scenery along each of the eight cycle paths. The program changes the speed of switching among the photographs according to the riding speed to simulate continuous motion. 


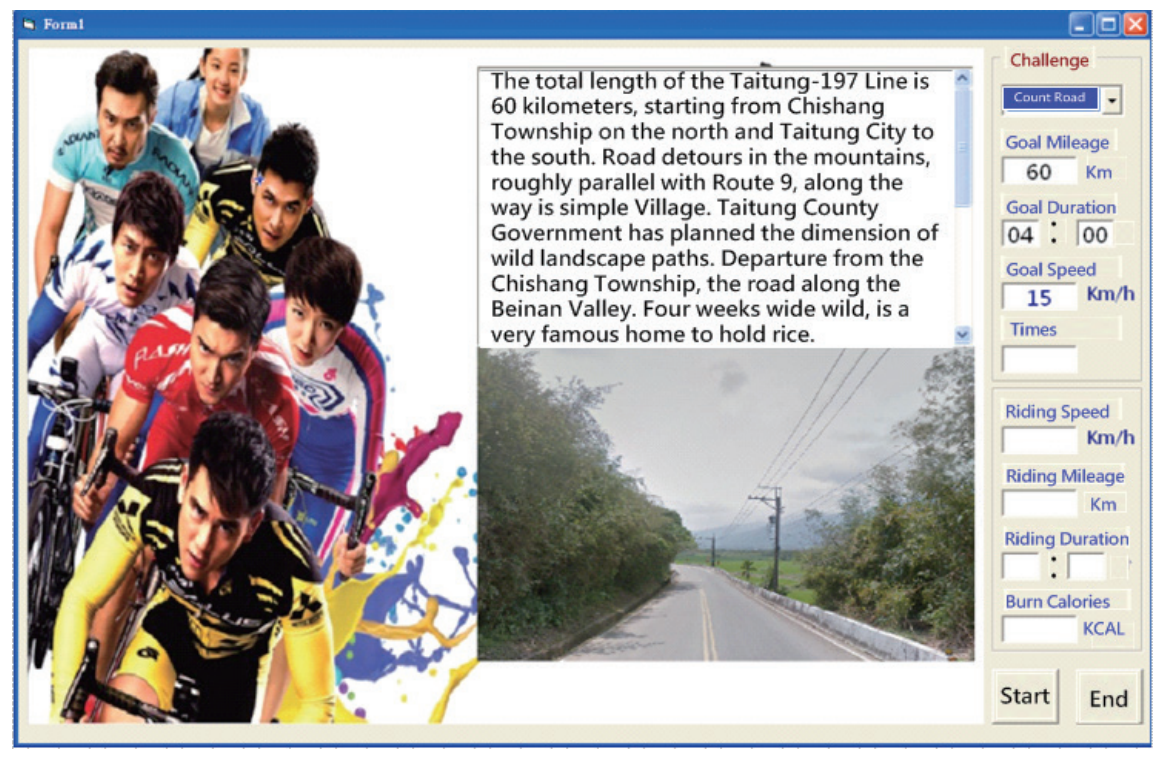

Fig. 7. (Color online) Selecting Taitung Chishang County Highway No. 197 as cycle path.

\section{Graphic Interface on Touch Panel}

The system uses an MT6071iE touch panel, developed by Weintek, to provide a humanmachine interface for a stationary spinning bike training device, which supports convenient operation. Figure 8 shows a photograph of the touch panel whose main specifications are as follows: (1) 7" backlight LCD panel, (2) $800 \times 480$ resolution, (3) five-wire resistive, (4) project files are downloaded using a USB Client, (5) equipped with COM1 (supporting RS232), COM2, and COM3 (supporting RS485) serial communication, (6) with protection marking of NEMA4/ IP65 and built-in power supply isolation, (7) 32-bit RISC Cortex A8 microprocessor with pulse frequency of $600 \mathrm{MHz}$, (8) $128 \mathrm{MB}$ flash memory (Flash) and $128 \mathrm{MB}$ dynamic memory (RAM), (9) built-in perpetual calendar, and (10) current consumption of $350 \mathrm{MA}$ at $24 \mathrm{~V}_{\mathrm{dc}}$.

The functions of the human-machine interface on the touch panel include the selection of the operational mode of the stationary spinning bike, selection of the cycle path, setting of training times, display of the simulated scenery, reading of client information from RFID membership card (including photographs and basic data), and reading of training information on RFID membership card (eight records in total). EasyBuilder Pro software provided by the Wintek touch panel includes a built-in graphic control tool box and it provides the function for users to write C-like macro instructions themselves. It is a program development platform with powerful functions. Figure 9 shows its window screen.

\section{Establishment of Experimental Platform}

To verify the feasibility of the stationary spinning bike training device herein, a stationary bike simulation platform was established in our laboratory. The outer size of the platform was $101 \mathrm{~cm}$ long by $110 \mathrm{~cm}$ high by $53 \mathrm{~cm}$ wide. The front wheel of the stationary bike was $16 \times 1.75$ 


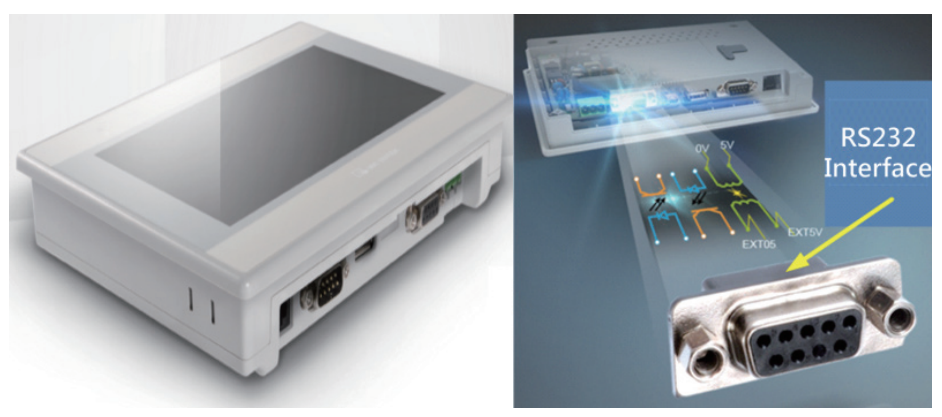

Fig. 8. (Color online) Photograph of MT6071iE touch panel.

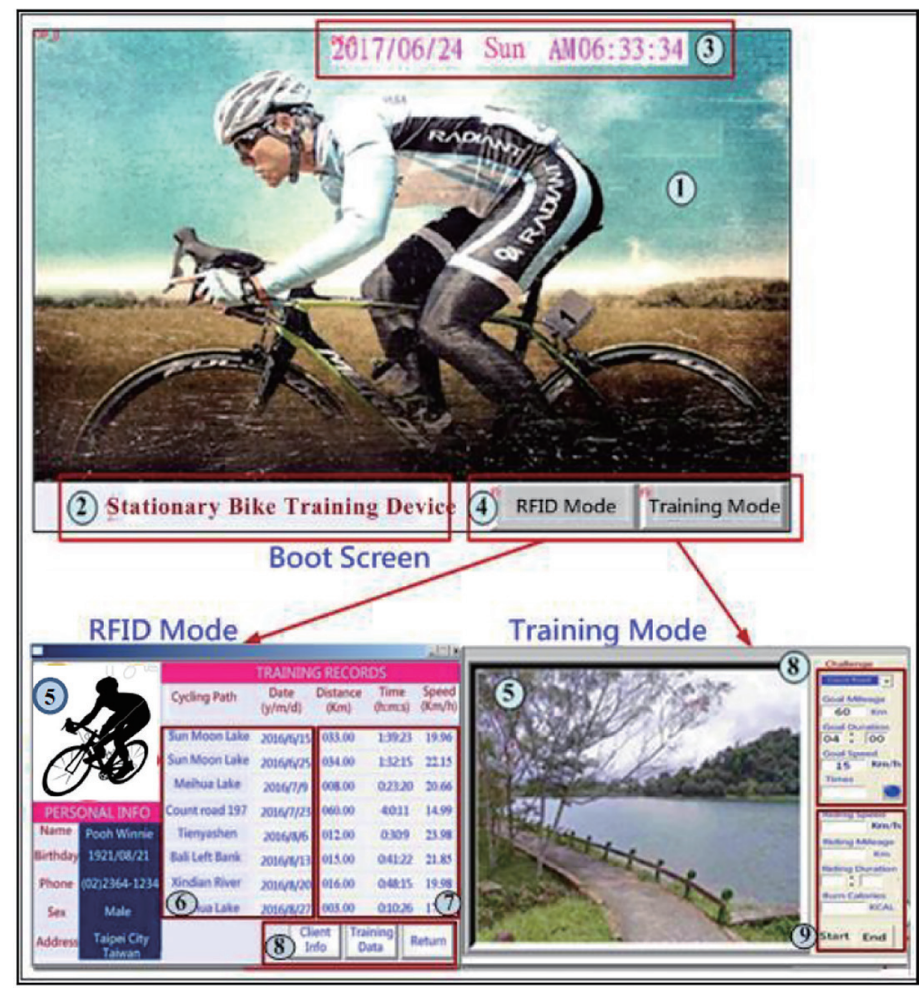

Fig. 9. (Color online) System consisting of the training mode and RFID mode.

inches, which represents a rim diameter of 16 inches and a rim width of 1.75 inches. The front wheel was attached to a brake caliper and a Hall sensor that was installed to detect wheel speed and supply this information to the odometer display.

The virtual reality training device was modified from an $18 \mathrm{~kg}$ stationary bike from San Sport.

(1) Installation of four magnets and Hall sensor

The electronic tachometer consisted of a Hall sensor and a ferrite ring, which was magnetized using the series method. $45 \mathrm{~N}$-poles and $45 \mathrm{~S}$-poles were thus generated. The surface magnetism was 350 gauss, and 45 signals were generated per revolution as shown in Figs. 10 and 11. Whenever the front wheel revolved, the Hall sensor sent out four pulses to the odometer, completing the establishment of the experimental device. 


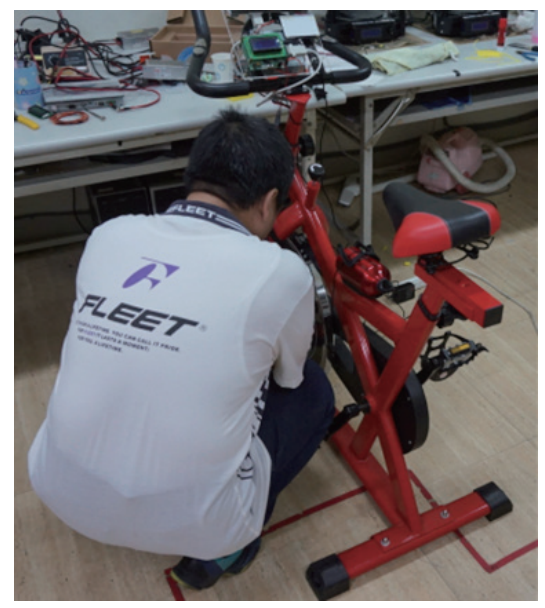

Fig. 10. (Color online) Hall sensor installation.

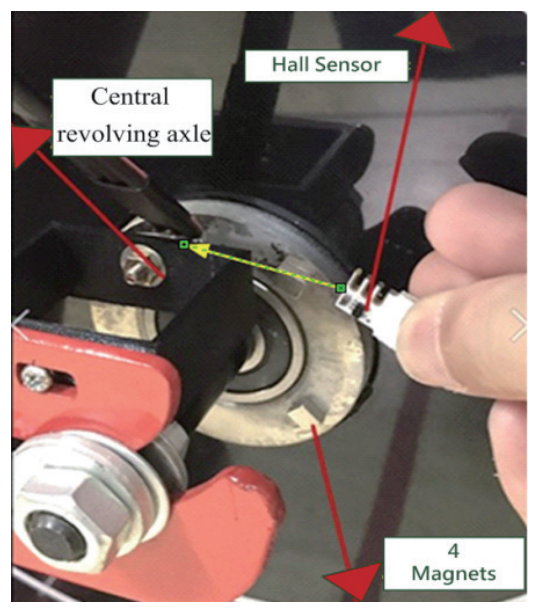

Fig. 11. (Color online) Hall sensor and four magnets.

(2) Development of software and hardware for odometer and testing of its function

Perform the welding and debugging jobs on the hardware circuit of the odometer and lay out the LCD module and odometer hardware circuit in the upper and lower combinations, as shown in Fig. 2.

(3) Program development and testing of simulation of Wintek touch panel

In this work, we designed the window screen and control program of the reality simulation with the graphic control interface on the Wintek touch panel. Serial communication components in the built-in graphic control tool box in EasyBuilder Pro software and the cycle executing element in the macro instruction are used in support of the function of the timer. The rider's average speed, accumulated mileage, and cycling duration will be calculated from the number of pulses that have been sent by the ATMega162 in the odometer to the Free Protocol in serial communication at each sampling time. The time to set up is $2 \mathrm{~s}$. All of the information will be displayed on the window screen. The rider's speed is used to determine the speed of photo-switching; a higher riding speed is associated with a high photo switching speed. Riders can therefore experience their movement through the scenery. Figure 12 shows the establishment of the experimental platform.

\section{Experimental Results and Function Testing}

The most important part of this work is the simulation. The riding speed affects the photoswitching speed in the program to simulate the continuous moving backward of the scenery. Figure 13 shows the actual landscape of the Sun Moon Lake Cycle Path. Figure 14 shows that of the Hsinchu Nanliao Fishing Port Cycle Path. Figure 15 shows that of the Liyutien Cycle Path. Figure 16 shows that of Taitung Chishang County Highway No. 197. Figure 17 shows that of the Tienyashen Cycle Path. Figure 18 shows that of the Bali Left Bank Cycle Path. Figure 19 shows that of the Xindian River Cycle Path. Figure 20 shows that of the Meihua Lake Roundthe-Lake Cycle Path. 


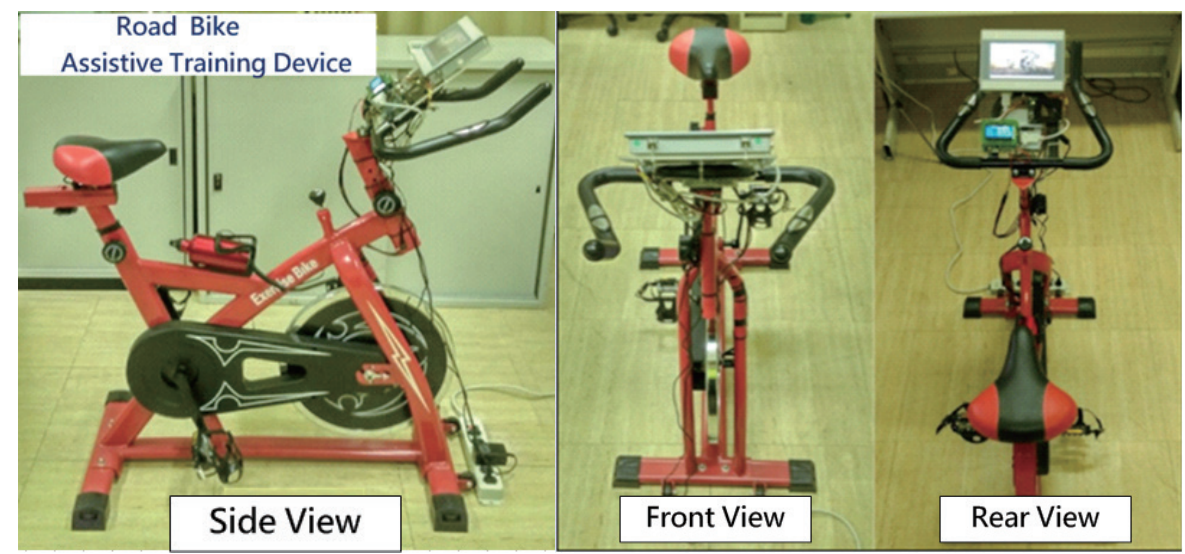

Fig. 12. (Color online) Establishment of stationary bike experimental platform.

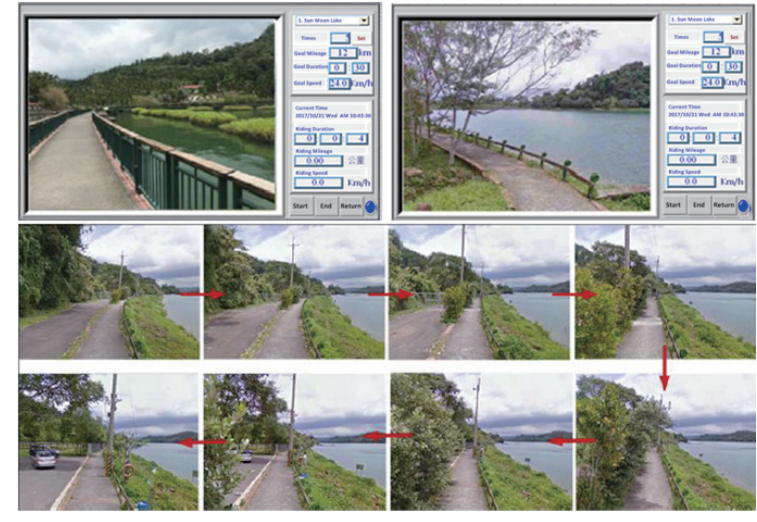

Fig. 13. (Color online) Actual landscape of Sun Moon Lake Cycle Path.
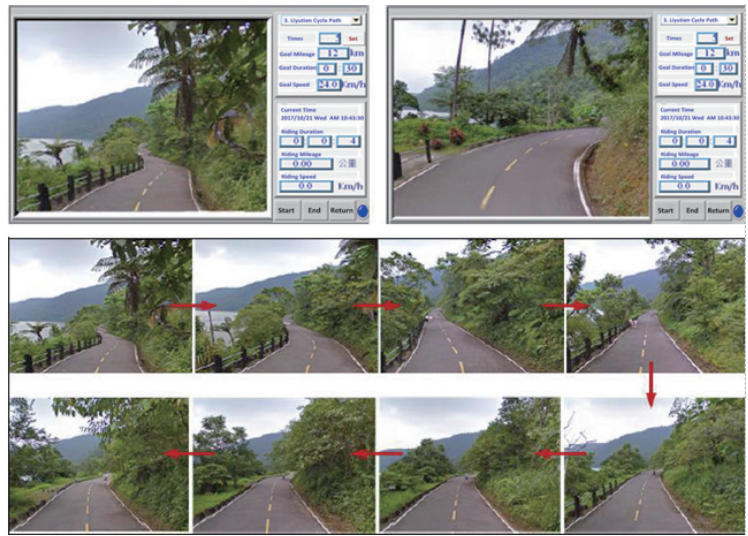

Fig. 15. (Color online) Actual landscape of Liyutien Cycle Path.

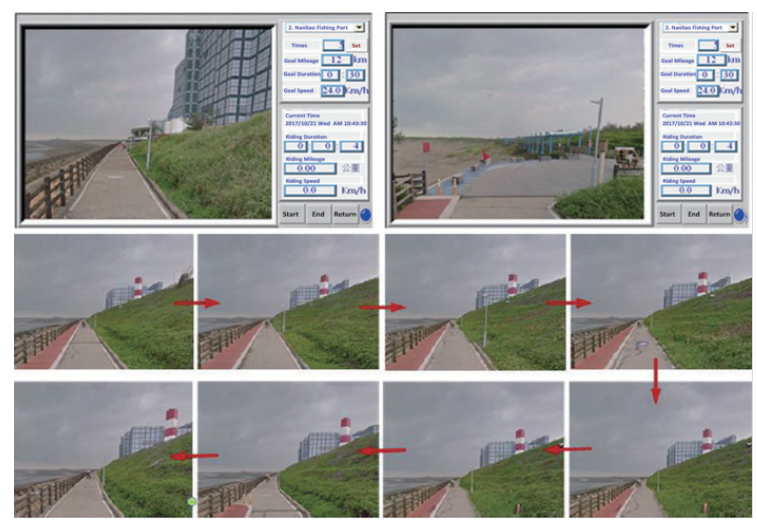

Fig. 14. (Color online) Actual landscape of Hsinchu Nanliao Fishing Port Cycle Path.
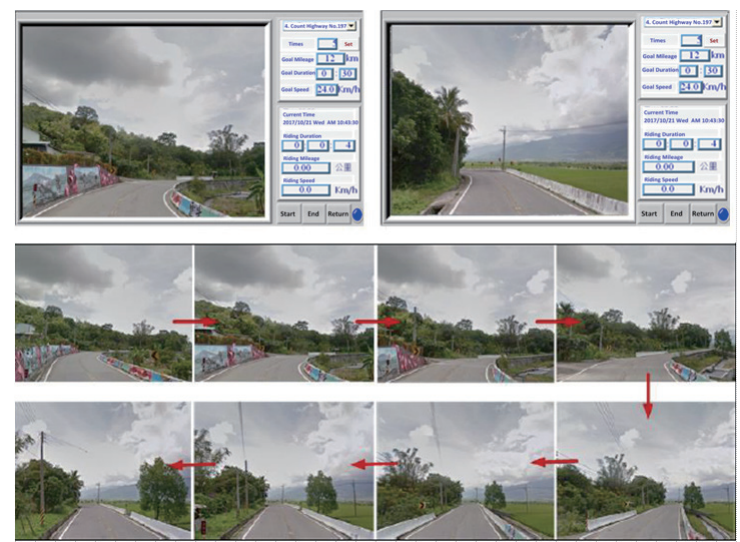

Fig. 16. (Color online) Actual landscape of Taitung Chishang County Highway No. 197. 

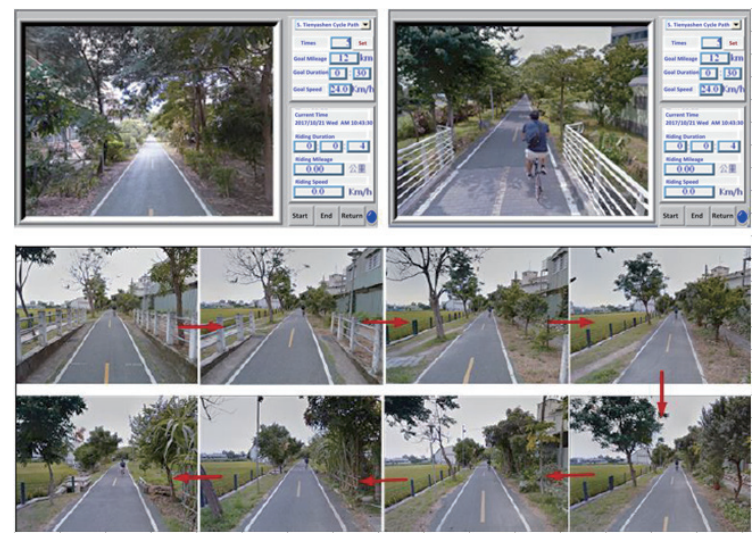

Fig. 17. (Color online) Actual landscape of Tienyashen Cycle Path.
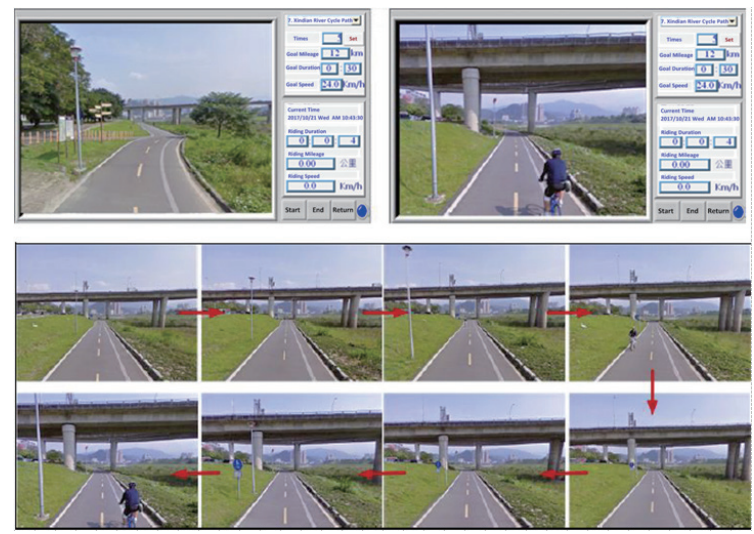

Fig. 19. (Color online) Actual landscape of Xindian River Cycle Path.

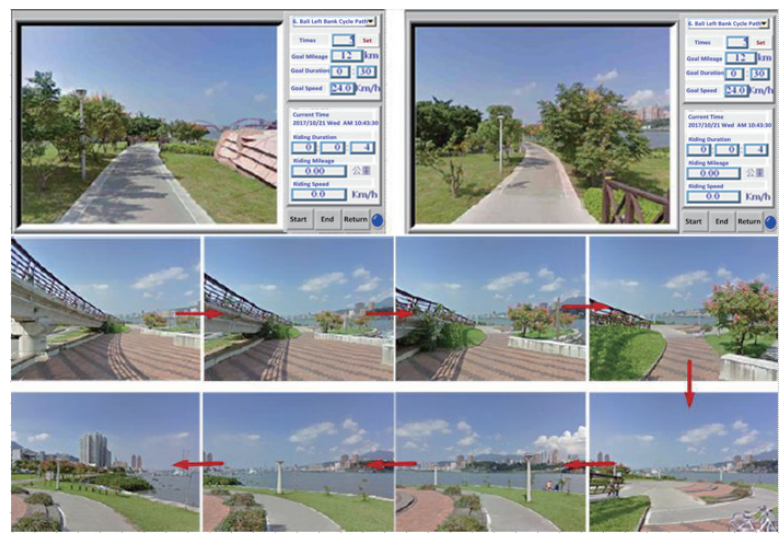

Fig. 18. (Color online) Actual landscape of Bali Left Bank Cycle Path.

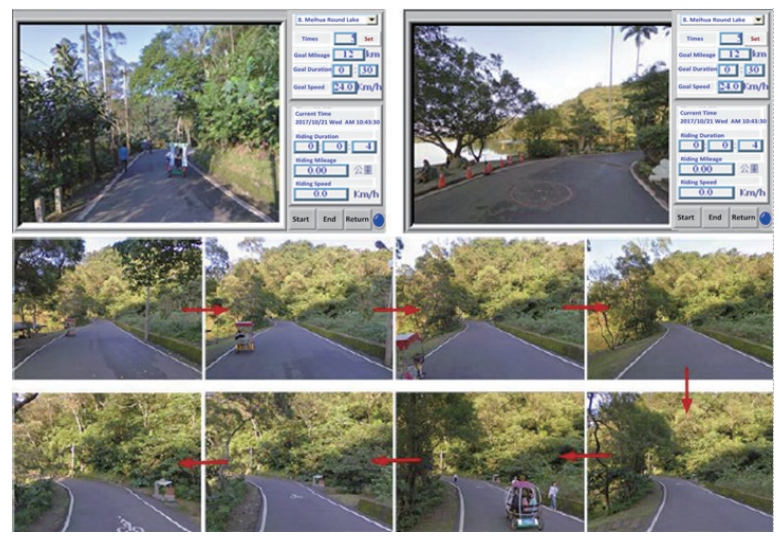

Fig. 20. (Color online) Actual landscape of Meihua Lake Round-the-Lake Cycle Path.

Once a rider has completed the training, he must put his personal training card (RFID membership card) on the RFID reader and press "End". The touch panel sends a signal to the odometer and the microcontroller inside to save the date, path selected, distance ridden, duration and mean speed (riding distance/duration) on the RFID card, comprising 10 bytes of information. The save format is as shown in the upper right-hand corner of Fig. 21.

When the touch panel is turned on, the screen displays the starting screen, "Road bike reality simulation training device". Two functional buttons are displayed in the bottom of the screen. The RFID mode can be selected, users can select "client info" or "traning data". The touch panel will then send an instruction to the microcontroller in the odometer, and displays the client information or training records of the member on the touch panel, as shown in Fig. 22. The rider's card in the figure contains eight training records, each of which includes path selected, training date, distance ridden, duration, and mean speed. Figure 22 clearly shows such data: the rider cycled on Taitung Chishang County Highway No. 197 on July 23, 2016, riding $60 \mathrm{~km}$ for $4 \mathrm{~h}$ and $11 \mathrm{~s}$, at an average speed of $14.99 \mathrm{~km} / \mathrm{h}$. 


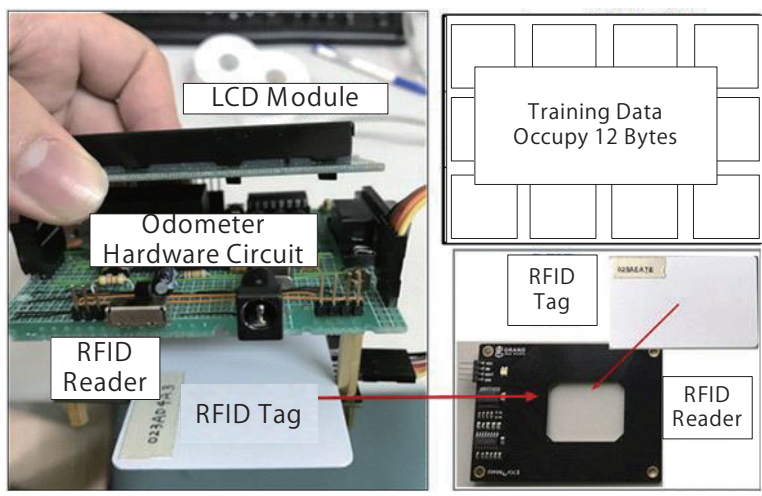

Fig. 21. (Color online) RFID card reading and data save format.

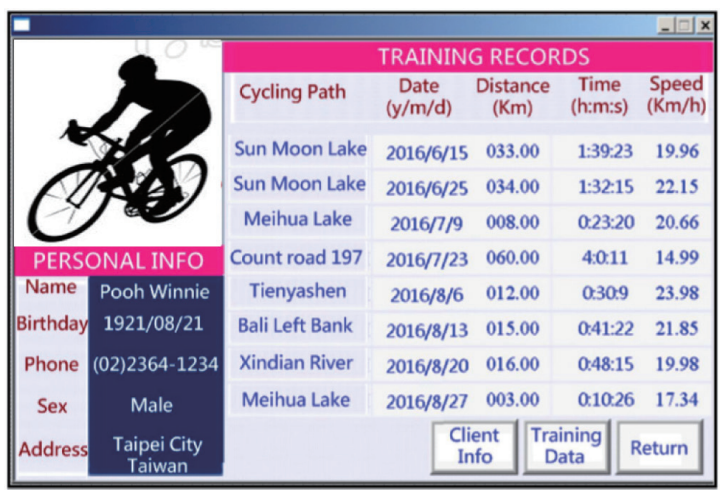

Fig. 22. (Color online) Eight displayed training records.

\section{Conclusions}

In this paper, we described the function development and design of the stationary bike reality simulation and the main goal is to make effort towards the increase in added value with the anticipation of modifying it into a real road bike training device in the future. We hope to add many innovative elements to road bikes, such as road bike APP intelligent program control design, road bike LED Bluetooth sound effect control and positioning system, road bike multi-functional dashboard camera, and a wireless communication system combined with an associated helmet accessory for riders. An integrated bike helmet uses a triple-axis accelerometer microsensor, combined with a smartphone, to perform the following functions. (1) The motion-detecting function of a triple-axis accelerometer can be used to measure the forward acceleration of the bike from the output voltage, and the forward acceleration and mileage can be estimated by the microcontroller; the calories consumed and duration of the exercise can be estimated from the weight of the rider, and all this information can be displayed on the odometer or a smartphone. (2) With the tilting detecting function of the triple-axis accelerometer, the head of the rider tilting to the right or left can be measured through the output voltage by the $X$-axis to control the LED lights on the left-hand and right-hand sides directly as direction indicator. (3) With LED bars on the top of the cycle helmet, a photoresistor senses the brightness of the environment; the LED bar can be activated in darkness to improve riding safety at night. (4) A flexible Bluetooth speaker is built into the bike helmet and can be used to listen to music that is played by the rider's smartphone, to receive global positioning system (GPS) navigation information from the smartphone, and in answering a phone call. (5) The collision-detecting function of the triple-axis accelerometer can be used to detect a collision of the bike or spillover during turning; when an abnormal physical situation is detected, the smartphone automatically calls for rescue.

\section{Acknowledgments}

The authors would like to thank the Ministry of Science and Technology, R.O.C., for financially supporting this research under Contract No. MOST 106-2511-S-197-001. 


\section{References}

1 R. Clingman and P. Pidcoe: IEEE Trans. Neural Syst. Rehab. Eng. 22 (2014) 879.

2 J. E. Zicker, W. J. Tompkins, R. T. Rubow, and J. H. Abbs: IEEE Trans. Biomed. Eng. 27 (1980) 509.

3 J. Mar, T. Y. Chang, and Y. J. Wang: Sensors 17 (2017) 1.

4 J. Zhang, W. Xiao, S. Zhang, and S. Huang: Sensors 17 (2017) 1.

5 M. Kim, C. Jeon, and J. Kim: Sensors 17 (2017) 1.

6 M. Koeva, M. Luleva, and P. Maldjanski: Sensors 17 (2017) 1.

7 S. Jayaram, H. I. Connacher, and K. W. Lyons: Comput. Aided Des. 29 (1997) 575.

8 F. Dong: Neural Comput. Appl. (2017) 1.

9 J. Peng, L. Xu, and Y. Shao: Neural Comput. Appl. 29 (2016) 1.

10 K. Elvedin, L. Robert, and I. I. Williams: J. Intell. Robot. Syst. 52 (2008) 79.

11 V. D. Lehner and T. A. DeFanti: IEEE Comput. Graph. Appl. 17 (1997) 13. 